\title{
Freeform wave-guiding and tunable frequency splitting in isotropic disordered photonic band gap materials
}

\author{
Weining Man ${ }^{1}$, Ying quan $\mathrm{He}^{1}$, Brian Leung ${ }^{1}$, Sam Tsitrin ${ }^{1}$, Marian Florescu ${ }^{2}$, Paul Steinhar $\mathrm{dt}^{3}$, Sal vatore \\ Tor quato ${ }^{3}$, Paul Chaikin ${ }^{4}$, \\ 1. Departm ent of Physics and Astronomy, San Francisco State University, San Francisco, CA 94132, USA \\ 2. Advanced Technology Institute and Department of Physics, University of Surrey, Guildford, Surrey GU27XH, United Kingdom \\ 3, Department of Physics, Princeton University, Princeton, NJ 08544, USA \\ 4, Department of Physics, New York University, New York, NY20012, USA \\ weining@sfsu.edu
}

\begin{abstract}
Using a new class of is otropic disordered photonic bandgap material, we demonstrate freeform photonics waveguides with arbitrary shapes and multi-channel frequency splitters with versatile tuning abilities, useful for signal proces sing and all-optical circu it applications.
\end{abstract}

(C)2012 Optical Society of America

OCIS codes: $130.5296,130.7408,160.5293,160.5298$

Inside photonic band gap (PBG) materials, light with selected frequencies can be confined and directed along chosen paths of line defects and around sharp corners [1], which is important for a large range of technological developments in telecommunication industry, laser engineering, optical computing, spectroscopy, etc [2]. However, in conventional PBG materials, periodic photonic crystals, only a limited number of rotational symmetries are allowed. The orientations of channels cut in photonic crystals for light guiding are strictly limited by the crystal symmetries, a disadvantage for potential applications.

Contradicting the long standing intuition that periodicity or long-range translational order is required in photonic band gap formation, a new class of disordered hyperuniform materials was predicted to possess sizeable and isotropic photonic band gaps [3]. Recently, we have experimentally observed isotropic PBG in these disordered materials [4]. In these isotropic disordered structures there are no preferential symmetry directions; hence it becomes possible to construct freeform channels with arbitrary shapes and bends in them [5]. We also demonstrated that line defects and point defects in these structures can be flexibly decorated to produce sharp resonant structures useful for filtering [5].

In terms of signal processing, photonic devices have many advantages over electronics, especially the large bandwidth. Since photons do not interact with each other, light signals of different frequencies can travel independently and simultaneously within the same channel. Hence, very compact frequency splitter becomes essential for large scale all-optical circuit applications. Since this new class of hyperuniform PBG material is isotropic and disordered, it provides the combined advantages of freeform direction choices not restricted by crystalline symmetries and flexible resonant tuning abilities. These advantages will enable the design of complex photonic circuits, for example, compact and tunable frequency splitters.

In this paper, we report the first experimental demonstrations of freeform wave-guiding through channels of arbitrary shapes, as well as tunable multi-channel frequency splitting of electromagnetic waves in 2D hyperuniform is otropic disordered PBG materials.

The 2D isotropic hyperuniform disordered PBG material constructed for this study consists of a network of $\mathrm{Al}_{2} \mathrm{O}_{3}$ cylinders, arranged at a hyperuniform disordered point pattern $[3,5]$. There is neither Bragg scattering nor long-range translational order in this structure. It was argued that hyperuniformity, combined with uniform local topology and short-range geometric order can explain the origin of PBGs in these disordered materials [3]. The PBGs are associated with local resonant scattering instead of Bragg scattering, hence are isotropic. Figure 1 shows finite difference time domain simulation results of electric field distribution of a guided mode through an "S" shaped channel created by removing cylinders along the curved path.

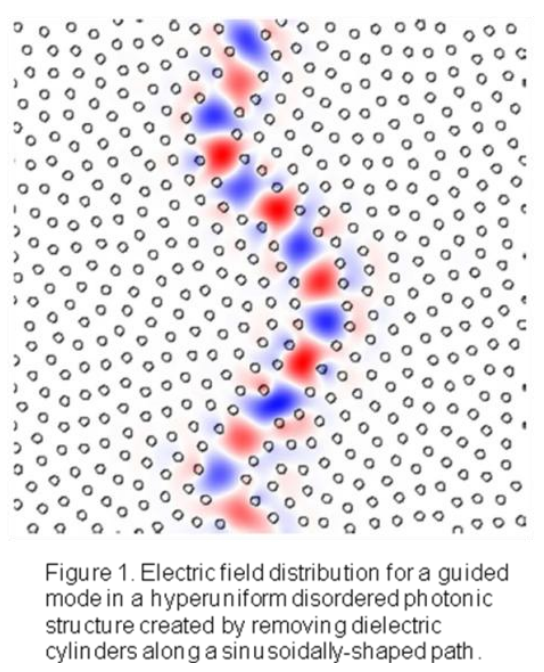


Experimentally, we used stereolithography to fabricate the bases of the structures at the scale of average spacing $a=13.3 \mathrm{~mm}$, and use commercially available $100.0 \mathrm{~mm}$ tall $\mathrm{Al}_{2} \mathrm{O}_{3}$ cylinders of radius $\mathrm{r}=2.5 \mathrm{~mm}$ and thin sheets of thickness $\mathrm{t}=0.38 \mathrm{~mm}$ of various width to assemble the hyperuniform network structure. Experimentally the photonic properties are measured using a HP-8510C vector network analyzer for microwaves with wavelength comparable to twice the cylinder spacing. The structure has a TM polarization PBG from 9.2 to $10.7 \mathrm{GHz}$ and a TE polarization $\mathrm{PBG}$ from 8.7 to $9.6 \mathrm{GHz}$. Wave-guiding channels are constructed and modified by removing rows of building blocks and adding individual extra defect cylinders. Transmission through the channels was measured by placing two microwave horn antennas right next to the channel openings. Absorption materials were placed around the sample to reduce noise.
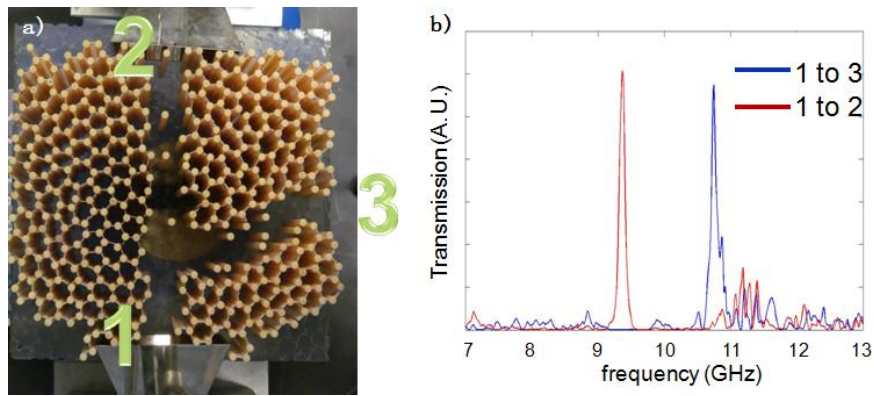

Figure 2. (a) A photo of a "Y" shape junction for frequency splitting. (b) Measured transmission through both output branches.
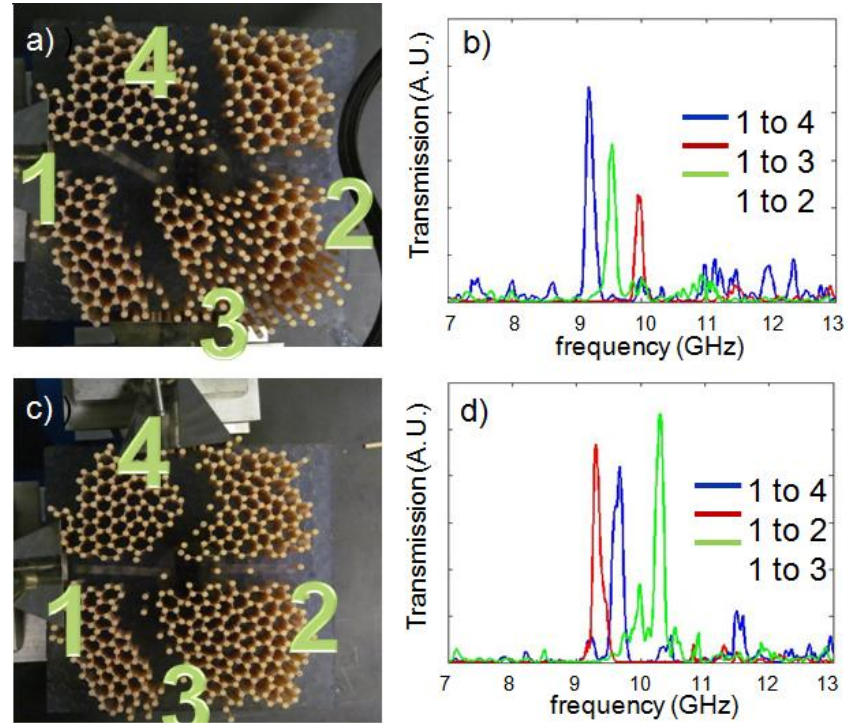

Figure 3. (a) \& (b) 2-step frequency splitting through a "tree" junction. (c) \& (d) 3-way frequency splitting through a "fork" junction.
Figure 2 shows a "Y" shape junction for frequency splitting. Continuous waves of different frequencies were sent into the input port marked as "1" on the photo. Transmissions are measured separately at two different output ports marked as "2" and "3", respectively. At the same time, signals of different frequencies are directed into different branches automatically. The disordered hyperuniform

PBG material offers a very flexible platform for defect design to select tunable frequencies, therefore the transmission peaks through two branches of the "Y" shape junction can be controlled and tuned by arranging different distribution of extra defect cylinders in the two output branches.

Figure 3 (a) and (b) show a two-step frequency splitter; while Figure (c) and (d) show a three-way frequency splitter. Again signals of different frequencies are automatically directed into different branches in various architectures. The propagating modes are strongly related to resonances built up inside the channel, enabling us to design local defects to control the passing frequency of each channel.

In summary, we have demonstrated novel architectures for freeform waveguides of arbitrary shapes, as well as compact frequency splitters with flexible tuning abilities, in isotropic PBG material. The ability to guide and split EM waves in a freeway format make this new class of disordered PBG materials good candidates for achieving highly flexible and robust platforms for integrated optical circuits.

This work was supported by the Research Cooperation and SFSU's startup fund.

[1] Shawn-Yu Lin etc. Science 282, 274 (1998)

[2] John D. Joannopoulos, etc. Photonic Crystals: Molding the Flow of Light (Princeton University Press, Princeton, 2008)

[3] Marian Florescu, etc. PNAS, 10649 20658-20663 (2009)

[4] Weining Man, etc. CLEO CThS2 (2010)

[5] Weining Man, etc. CLEO QF3H2 (2012) 